\title{
Health-Related Quality of Life in Heart Failure in Jordan from Patients perspectives
}

\author{
Haneen M. Abu Hayeah ${ }^{1}$, Ahmad R. Saifan ${ }^{2}$, Mohannad E. AbuRuz ${ }^{3}$, \\ Mohannad A. Aljabery ${ }^{4}$ \\ ${ }^{1}$ (Clinical Nurse Specialist, ministry of health, Jordan) \\ ${ }^{2}$ (Associate professor, Department of adult health and critical care, Faculty of Nursing, Fatima College for \\ Health Science, Abu Dhabi, UAE) \\ ${ }^{3}$ (Associate professor, Department of clinical nursing, Faculty of Nursing, Applied Science Private University, \\ Jordan) \\ ${ }^{4}$ (Clinical Nurse Specialist, ministry of health, Jordan)
}

\begin{abstract}
Background: Heart failure is a global burden and a major public health issue, especially in developing countries, including Jordan. Most related research was conducted in Western cultures and may have limited applicability for individuals in Jordan. Aim: To explore the quality of life of patients with heart failure in Jordan from their perspectives. Methods: A qualitative descriptive design was adopted. Semi-structured interviews with 25 heart failure patients were undertaken. Results: Analysis of transcripts identified three major themes. The first theme described the awareness of health related quality of life (HRQoL) concept and perceptions of patients about heart failure $(H F)$. The second theme described the influence of HF on patients in terms of physical ability, psychosocial life, spirituality, economic status and cognitive aspects. The third theme showed different steps to improve HRQoL from patients' perspectives. Conclusion: HRQoL is a multidimensional, subjective concept that is affected by a variety of factors. Heart failure had negative impacts on the patients' HRQoL, especially in the physical domain.
\end{abstract}

Keywords: Ejection fraction, Heart failure, Jordan, and quality of life

\section{Introduction}

Heart failure (HF) is a global burden, with 23 million patients all over the world, and strong indications of rising [1], with one estimate predicting an increase of $25 \%$ from 2013 to 2030 [2]. In the US more than 5.7 million people have HF, and more than 670,000 new HF patients are diagnosed yearly, costing about $\$ 33.2$ billion annually[3-4]. Around 10\% of French patients over 59 years have HF [5]. In developing countries, the number of HF patients is rising. For instance, in Saudi Arabia there are an estimated 455,222 cases, and 32,200 new cases per year [6]. In Jordan, a developing Arabic country with an estimated population of 5.61 millions, the prevalence of HF is approximately 100000 ; the estimated incidence is 8251 annually [7].

The World Health Organization (WHO,1995, p1403) defined quality of life (QoL) as "Individuals' perception of their position in life in the context of the culture and value systems in which they live and in relation to their goals, standards, and concerns" [8]. Health related quality of life (HRQoL) is considered to be a more focused view of the QoL concept, in which issues not directly related to health are excluded, such as housing, neighborhood and economics [9].

Heart failure patients reported poor HRQoL in different studies $[3,6,10]$. Most studies on HF in general, and particularly on HRQoL for HF patients, were conducted in Western countries, with more than thirteen studies being conducted in the US, France and Germany alone. However, there is a paucity of information about HRQoL for HF patients in developing countries, and a major lack for Arab countries such as Jordan. Acute myocardial infarction, cerebrovascular accidents and cancers are the leading cause of death in Jordan [11]. The population in Jordan is estimated around 9.5 millions including citizens and refugees. Among those, 5\% are 60 years old and above [12]. Elderly people are more susceptible to high blood pressure, diabetes, and high blood cholesterol, all of which are risk factors in cardiovascular diseases [13]. The risk factors that may contribute to the cardiovascular disease in Jordan are obesity, diabetes and asthma [11, 14].

Heart failure is the final result of cardiovascular diseases [15], and an increasing proportion of the Jordanian population is susceptible to the disease simply due to increasing age, as well as lifestyle factors; the number of HF patients is expected to increase dramatically in the coming years. As noted previously, HRQoL in general has been widely studied, but it has not been applied to the case of HF patients in Jordan. Furthermore, the predominantly Western context of most existing studies means many of their findings might or might not be applicable the Jordanian context; therefore this study addresses an important research gap by exploring in depth HRQoL among HF patients in Jordan. 


\section{Design, Sample and Setting}

\section{Methodology}

A qualitative descriptive design was adopted. Semi-structured interviews were held in three hospitals in Jordan. A purposive sample with total of 25 patients was recruited from the cardiac outpatient clinics. The inclusion criteria were a definitive diagnosis of HF. Their ages were more than 18 years, and lived in Jordan. Severity of HF measured by ejection fraction (preserved ejection fraction more than $40 \%$, and low ejection fraction below 40\%) [16]. Participants were in stable health status at the time of interviews. The participants who had any other chronic diseases that might affect HRQoL such as cancer, psychiatric disorders, renal failure, or liver transplantation were excluded. .

\section{Ethical consideration}

The study was approved by the IRB committee at the Applied Science Private University and all hospitals were data collection was performed. Prior to interviews participants signed a consent form after a though explanation about the aims and the procedure of the study.. All participants were free to participate and they had the right to withdraw from the study any time they wished. Participants' identities remained confidential by replacing numbers instead of their real names. The records were kept in password-protected computers accessible only to the principle investigator and research team.

\section{Data analysis}

The recorded interviews were transcribed, and translated back in English, since the spoken language in Jordan is Arabic. Thematic analysis NVivo software (version 11) was used in order to interpret the data

\section{Results}

Data collection took place between February and March 2016. The sample were 13 male and 12 female aged between 26 and 88 years (mean 63 years), with varying severity as measured by ejection fraction. The participants' level of education varied from not educated to bachelor degree. All were found to have co morbidities hypertension, diabetes, coronary artery disease, and hyperlipidemia except of two participants their cardiac condition was worsened because of pericarditis. Additionally they were all smokers except of two participants. The detailed description of sample is shown in table 1.

The transcripts were read repeatedly and were arranged into initial codes emerged inductively, and then developed to produce major themes. Three major themes were identified as shown in table 2 . The most popular definition of HRQoL was the ability to do daily living activities including physical and social activities. Most of the participants considered HRQoL as being able to do the daily activities by their own without help, like they used to do before getting sick. "..Quality of life means that I can do what I used to do every day without help...". Some participants focused on psychological domain such as being happy and satisfied with living. These participants explained that physical limitations are not important to reduce HRQoL. Some of these talked about cases from the reality such as some successful persons who have physical handicapped. However a few participants stated that happiness is not important to be related to physical or mental wellbeing. They thought that economical domain is more effective in presence or absence of happiness which would be reflected on QoL. ".. I can say that good QoL includes being happy most of the time..". "..Oh, for sure money is what your life stands for.. actually it is the first thing came to my mind when you say $Q o L "$.

Some participants provided another definition of HRQoL which includes fulfilling relationships. They expressed that having a good QoL is related to have family and friends in around to take care of you, and doing what you want to do with the people you love. "What do you mean by QoL? ... I guess the most important issue that you enjoy the life with your family. Family bonds, friends' support this is what you call it a good life...".

Most of the participants gave a description of HF as having a weak heart associated with fluid collection in the lungs which results in shortness of breath. However, they had not specific medical information about heart failure. It was clear that most of the interviewees obtained their definitions from their experiences but not from reading or education from healthcare providers. "...All what I know is that I have a sick heart..". "..Actually I did not read anything about it..".

Surprisingly, most of the participants responded by accepting their condition. These participants explained that they suffered from some health problems earlier. Therefore, they were not surprised when diagnosed with HF and they started to think how they will deal with their disease. However, few participants denied that they have any cardiac problem. They expressed that their signs and symptoms such as shortness of breath were resulted from chest infection. ".... I told them that I did not have HF and my heart was fine, it is just my chest...". In addition, some of the participants compared themselves to others, especially with whom in the same age. ".... Why this happened to me, I am still young... ". Some of them wondered why this would happen to them. Some of them gave examples from the real life about friends or relatives who used to smoke or used to have stressors in their life and none of them was diagnosed with HF. On the other side, some of the participants 
completely believed that their disease was a test from God. They explained that all diseases are from God and they challenge people's faithfulness. "I am always thankful for God, by taking something valuable from us, God tests our patience and faithfulness....".

The negative impact of HF on physical ability was the most common topic expressed by the participants. When the participants were asked to describe their conditions, the most common symptoms were mentioned included difficulty in breathing accompanied with cough, edema, and fatigue which had negative impacts on their QoL. One of the participants stated that she suffered most of the times from difficulty in breathing and cough. This limited her daily activity living. This situation was considered by most of the participants as a reason to worsen their QoL

Most of the participants said that their social activities were affected negatively in a remarkable way. For example, some of the participants talked about how they cannot visit their friends, going out with their families, or shopping anymore as they used to. However, some of them decreased the influence of HF on their social side. They were more neutral and stated that there were no differences in social activities as they did not consider themselves sick. In contrast, few participants mentioned that their social activities improved after being diagnosed with HF. These participants stated that they start to have more times to be spent with their families. The criticality of their conditions was viewed as a reason of getting compassion of their relatives and friends. This led many people around them to strengthen their mutual relationships by increasing the visiting numbers and times.

Spirituality was identified as a crucial issue by most of participants, which had a positive effect on HRQoL. Spiritual life of participants expressed in faith in God, reading the holy books, and praying. Furthermore, some participants included other activities in their spiritual life such as listening to music and relaxation. "... Praying and reading Qur'an are activities make us closer to the God and strengthen our hearts so we can face most of life problems....".

Changes in sleeping pattern, forgetting to take medications and changes in concentration were addressed as HF impact on cognitive functioning. Several participants reported changes in sleeping patterns or difficulty in initiating sleeping. " "... Most of the times, I cannot sleep at night because of cough, I feel myself suffocated...". Forgetting to take medications was an issue mentioned by some participants which affected their health condition negatively. "... I have to take a lot of bills, I have hypertension, diabetes, high cholesterol and the medications of HF..". Moreover, nearly all participants reported that their health condition had negative impact on their concentration. Some participants mentioned that they have a lot to think and worry about so they cannot focus on things they used to before. Some participants relayed this concern to the bad quality of sleeping they have, while others to aging. "... I cannot focus on everything ... I am exhausted, fatigue and not sleeping very well..".

Furthermore, economical status was a great concern of the participants because of losing jobs and health care expenses especially with recurrent admissions. "... My health got worse, my heart become weaker... I cannot work anymore..". Most of the participants reported bad financial situation especially men, even though they were covered by public or private health insurance. However, six participants were not covered by any insurance and they pay the treatments expenses from their own money. Significantly, even the participants who have health insurance, they complained that some of the treatments or medications were not covered by insurance. All the participants stated that recurrent admissions are a burden economically and psychologically.

The participants highlighted some strategies they thought to improve their QoL. Most of the participants explained that taking a small walk and fresh air could help in reliving symptoms and feeling better. "... When I feel suffocated, I sit in my house backyard garden ... this make s me feel better...". Also, majority of the participants focused on maintaining the health related behaviors such as adherence to medications, low sodium diet, healthy diet, quit smoking and treating disease symptoms. "... I know that quitting smoking, eating low salt diet improve HF patients and decrease symptoms..". However, some of the participants made changes in their houses or left to another house that suited their health condition. "... I had to move out to another house because of stairs..".

Social support was thought to have positive impacts on participants' HRQoL. "... To find someone looking for you and care about you is the most important thing make you overcome the challenges of the disease..". Spiritual activities were addressed to have positive impacts on HRQoL and helped the participants for more acceptance of the disease as mentioned earlier. Finally, some participants mentioned that there should be a special supportive treatment to help HF patients to continue in their career. The others suggested that HF patient should retire and the government should have a specific system for HF patients to help them economically. 


\section{Discussion}

Participants' perceptions assured the subjective, compound nature of HRQoL. The definitions of HRQoL expressed the impact of HF on their daily activities, also the pursuit of happiness and maintaining relationships. Moreover, participants reported negative impacts of HF on their QoL in multiple domains such as physical, psychosocial, spiritual, cognitive, and economical dimensions. Furthermore, steps to improve QoL were mentioned.

Most definitions of HRQoL implied its subjective nature [17-20]. Scott et al, definition of HRQoL expressed its subjective nature: "the overall effect and outcome of an illness and its treatment on an individual's physical, psychological, and social well-being as perceived by that individual (p. 84)" [19]. In the same manner Alla et al, defined HRQoL as "a multidimensional concept, based on the patient's own perception of his health which integrates not only the functional or physical dimensions of the disease, but also psychological and social dimensions (p. 337)" [18]. The definitions of HRQoL given by participants in the current study were similar to those mentioned earlier. However, the participants described their willing to have a betterQoL even with the negative influence of their health condition.

The definitions of HRQoL included three components: the ability to do physical and social activities, the feeling of happiness, and maintaining relationships with the significant others. The most common definition mentioned by participants was the ability to do physical and social activities. Many studies reported that physical capability is a significant component of HRQoL [21-23]. This is may refer to the significance of physical daily activities as it was related to their social roles. Other definitions implied the psychological condition in pursuit of happiness and in maintaining relationships. Several studies encompassed psychosocial status and wellbeing as a crucial domain in HRQoL [23-25].

Participants in general gave a description of HF and common symptoms such as shortness of breath due to fluid collection similarly to another study [26]. Dyspnea and fluid retention are considered as main symptoms of HF [3, 27-29]. Even though participants expressed a general view of HF depended on their experiences but not from reading or health education. This may referred to difficulties in communication with the physicians and nurses as stated by participants in agreement with the findings of other study [26]. However, some participants thought that extra of knowledge and deep information about the disease may increase the level of stress contradicting other studies. Effective discussion about disease prognosis showed benefits to cancer patients [30], HF showed worse prognosis than many cancers [31-33].

Heart failure is a final and irreversible result for many heart disorders. Therefore, hypertension, coronary artery disease, and cardiomypathy may cause HF [15]. Diabetes and obesity are considered as major risk factors, while smoking, high fat diet, infection and stress considered as minor risk factors [34]. This is matched the characteristics of the sample in the current study as all participants were found to have HTN, DM, $\mathrm{CAD}$, and hyperlipidemia except of two participants their cardiac condition was worsened because of pericarditis. Additionally they were all smokers except of two participants. It is worth mentioning that most of the participants mentioned smoking and stress as reasons for HF, while few of them gave the right reasons. This is may refer to their general knowledge about HF as mentioned earlier, even though they knew that these factors lead somehow to HF.

The findings revealed about how participants' HRQoL domains were affected by HF, which were the core of the current study. Most of the participants reported that they have physical symptoms associated with HF such as dyspnea, fatigue, and edema. In agreement with prior studies reported that main symptoms of HF are dyspnea, fatigue, fluid retention, and pulmonary congestion [27-28, 35]. Therefore, physical symptoms are burden and have negative impacts on HRQoL $[29,36]$. Most of the participants reported negative impacts on their physical ability similarly to prior studies. However, only two participants reported minimal negative physical limitations. This may refer to the relatively short period since diagnosis, and having a preserved ejection fraction of above $40 \%$. Patients with preserved ejection fraction have lower mortality rate and hospitalization recurrence more than patients with low ejection fraction [37]. Ejection fraction above $40 \%$ tends to be considered as preserved ejection fraction [16].

In relation to psychosocial life participants reported changes in social activities and relationships, also expressed negative emotions like anxiety and frustration toward their health condition. Some participants revealed about negative impacts of HF on their social activities and relationships. This may due to physical limitations, recurrent hospitalizations, financial status and the feeling of dependency on family members. This is consistent with the result of prior studies that HF has negative impacts on psychosocial life of the patients [3839]. In addition, HF interferes to the physical, social status, and increased distress [40]. Furthermore, participants expressed negative emotions similarly with other study reported that $30-40 \%$ of patients with HF have emotional distress such as depression [41]. Depression and anxiety are parallel status for HF population, which reflects poorer HRQoL and reduction in physical activity [27, 31, 35].

Therefore, physical and emotional symptoms impact on HRQoL should be assessed by researchers and clinicians. There are many instruments to assess HF symptoms to provide a proper intervention according to 
assessment and its impact on QoL. For example, The Dyspnea-Fatigue Index includes 4 items measuring dyspnea and fatigue for HF patients and their impact on daily activities [42]. Also the Beck Depression InventoryII or the Patient health Questionnaire-9 to assess the depressive symptoms [43-44]. Moreover, home health care programs and health education may improve physical and emotional status, financial condition, and QoL [45-46].

However, some participants contradicted the results that mentioned earlier. They reported positive impacts on their social activities and relationships, this may due to several reasons. This may due to social support as shown it is beneficial to health outcomes and improved QoL [6]. One of the Islamic and Arabic world features is extended families who provide physical, psychological, and social support to the individual especially in illness [47]. In relation to Islamic and Arabic culture beliefs the individuals' significant others make concerted efforts to provide support for patients in order to get rewards from God and meet social obligations [47].

Participants in the current study reported that HF influenced their economical status negatively due to job loss and recurrent hospitalization or increase medical expenses. This is in agreement with prior studies reported that HF patients with recurrent admissions, may suffer economically and this will have negative impacts on their QoL [48-49]. Most of the participants reported that they lost their jobs due to physical symptoms. It is worth mentioning that men were affected more than women economical wise, this may refer to the nature that man takes responsibility of the family especially financially [50-51]. Therefore, further studies are recommended to provide effective support for those who suffer economically.

Some participants expressed that their cognitive aspects were affected negatively by HF such as forgetting to take medication, low concentration, and sleeping difficulties. This is consistent with the result of a prior study reported that cognitive dysfunction is prevalent among HF population [38]. Treatment of multiple medications and prolonged duration of HF reflect poorer HRQoL [34]. The participants in the current study their duration of diagnosis of HF ranged from 2-12 years as this may explain some of the poor results of HRQoL.

Sleeping disturbances has been reported in HF patients. Initiating sleep, maintaining sleep, and early morning awakening play an important role in low HRQoL [52]. Some participants expressed sleeping disturbances which is consistent with a prior study. In the same manner, poor quality of sleeping may affect the cognitive functions as a result, and this may lead to poorer QoL [33].

Spiritual activities such as faith in God, praying, reading holy books, and listening to music have been shown to have positive impact on HRQoL [39]. Spirituality explained $24 \%$ of the difference of HRQoL worldwide in HF patients [53]. Islamic and Arabic culture can have an important role on an individuals' health [54]. Participants in the current study expressed that faith in God and spiritual activities helped them in accepting their health condition and provided them with internal relief. However, some of the spiritual activities were negatively affected due to the limitations in physical ability.

Finally, the participants mentioned ways to improve the HRQoL. Many studies talked about the importance of exercise and physical activity and its positive impacts on HRQoL [27-28, 48]. In addition, adherence to pharmacological and non pharmacological treatment has positive impacts on HRQoL in HF patients [55]. Moreover, dieting such as low salt diet, low fat diet, fruits and vegetables may improve the HRQoL [56]. Also, social support and spirituality have been shown to improve HF patients QoL as mentioned earlier.

\section{Conclusion}

HRQoL is a multidimensional, subjective concept that is affected by a variety of factors. Heart failure had negative impacts on the patient's HRQoL especially in physical domain. The current study is the first of its kind in Jordan. This study gained strength from many reasons. The first one was the subjectivity of the results as well as HRQoL is subjective concept reflects each patients' point of view. The second reason was the sample size is relatively large when compared to other studies. Finally, the results included a comprehensive analysis of all HRQoL dimensions, which added more reliability of it.

\section{TABLES}

Table 1: Description of the sample

\begin{tabular}{|c|l|c|c|c|c|c|c|}
\hline$\#$ & Gender & Age & Duration of & EF & co morbidities & Smoker & Previous admissions \\
\hline 1 & Male & $58 \mathrm{y}$ & $6 \mathrm{y}$ & $34 \%$ & HTN, Aortic dilatation & Yes & 15 \\
\hline 2 & Male & $61 \mathrm{y}$ & $5 \mathrm{y}$ & $30 \%$ & HTN, DM & Yes & 10 \\
\hline 3 & Male & $48 \mathrm{y}$ & $5 \mathrm{y}$ & $30 \%$ & HTN, DM & Yes & 20 \\
\hline 4 & Male & $26 \mathrm{y}$ & $3 \mathrm{y}$ & $18 \%$ & Infection & Yes & $40-60$ \\
\hline 5 & Male & $55 \mathrm{y}$ & $7 \mathrm{y}$ & $35 \%$ & HTN, CAD & Yes & 30 \\
\hline 6 & Male & $63 \mathrm{y}$ & $6 \mathrm{y}$ & $30 \%$ & HTN, DM & Yes & 27 \\
\hline
\end{tabular}


Health-Related Quality of Life in Heart Failure in Jordan from Patients perspectives

\begin{tabular}{|c|c|c|c|c|c|c|c|}
\hline 7 & Male & $74 y$ & $4 y$ & $40 \%$ & HTN, CAD & Yes & 10 \\
\hline 8 & Male & $80 \mathrm{y}$ & $8 y$ & $35 \%$ & HTN, DM, CAD & Yes & 40 \\
\hline 9 & Male & $67 \mathrm{y}$ & $2 y$ & $42 \%$ & HTN, CAD & Yes & 5 \\
\hline 10 & & $75 y$ & $10 \mathrm{y}$ & $30 \%$ & HTN, DM, CAD & Yes & $40-50$ \\
\hline 11 & Female & $35 \mathrm{y}$ & $2 \mathrm{yrs}$ & $40 \%$ & Pericarditis & No & 12 \\
\hline 12 & Female & $55 \mathrm{y}$ & $3 y$ & $42 \%$ & HTN, hyperlipidemia & Yes & 10 \\
\hline 13 & Female & $80 \mathrm{y}$ & $10 \mathrm{y}$ & $25 \%$ & HTN, DM, CAD & Yes & $50-70$ \\
\hline 14 & Female & $72 y$ & $7 y$ & $30 \%$ & HTN, DM & Yes & 20 \\
\hline 15 & Male & $56 \mathrm{y}$ & $4 y$ & $35 \%$ & HTN, CAD & Yes & 10 \\
\hline 16 & Male & $71 \mathrm{y}$ & $8 y$ & $25 \%$ & $\begin{array}{l}\text { HTN, DM, CAD, } \\
\text { hyperlipidemia }\end{array}$ & Yes & 30 \\
\hline 17 & Male & $66 \mathrm{y}$ & $5 y$ & $35 \%$ & HTN, CAD & Yes & 10 \\
\hline 18 & Male & $49 y$ & $2 y$ & $40 \%$ & HTN & Yes & 6 \\
\hline 19 & Female & $58 \mathrm{y}$ & $4 y$ & $42 \%$ & HTN, DM, obese & No & 13 \\
\hline 20 & Female & $61 \mathrm{y}$ & $9 y$ & $30 \%$ & HTN, DM, CAD & Yes & 50 \\
\hline 21 & Female & $59 y$ & $3 \mathrm{yrs}$ & $35 \%$ & HTN, CAD & Yes & 10 \\
\hline 22 & Female & $70 y$ & $12 y$ & $25 \%$ & $\begin{array}{l}\text { HTN, DM, CAD, } \\
\text { hyperlipidemia }\end{array}$ & Yes & $>60$ \\
\hline 23 & Female & $67 y$ & $7 y$ & $35 \%$ & HTN, DM & Yes & 20 \\
\hline 24 & Female & $81 \mathrm{y}$ & $10 y$ & $25 \%$ & $\begin{array}{l}\text { HTN, DM, CAD, } \\
\text { hyperlipidemia }\end{array}$ & Yes & $>40$ \\
\hline 25 & Female & $88 \mathrm{y}$ & $8 \mathrm{y}$ & $35 \%$ & $\begin{array}{l}\text { HTN, DM, CAD, } \\
\text { hyperlipidemia }\end{array}$ & Yes & $>50$ \\
\hline
\end{tabular}

HTN: Hypertension, DM: Diabetes mellitus, CAD: Coronary artery disease.

Table 2: Identification of themes

\begin{tabular}{|c|c|}
\hline Subthemes & Themes \\
\hline $\begin{array}{l}\text { 1. Patients' understanding of HRQoL. } \\
\text { 2. Knowledge about HF as a disease. } \\
\text { 3. The first response when receiving the diagnosis of HF. }\end{array}$ & $\begin{array}{c}\text { Awareness of HRQoL and perceptions } \\
\text { about HF. }\end{array}$ \\
\hline $\begin{array}{ll}\text { 1. } & \text { Physical ability. } \\
\text { 2. } & \text { Psychosocial life. } \\
\text { 3. } & \text { Spirituality. } \\
\text { 4. } & \text { Cognitive aspects. } \\
\text { 5. } & \text { Economical status. }\end{array}$ & Influence of $\mathrm{HF}$ on patients. \\
\hline $\begin{array}{ll}\text { 1. } & \text { Take a short walk and obtain fresh air. } \\
\text { 2. } & \text { Health related behaviors. } \\
\text { 3. } & \text { Housing adjustments. } \\
\text { 4. } & \text { Social support. } \\
\text { 5. } & \text { Spiritual activities. } \\
\text { 6. } & \text { Job reconsiderations regarding health status. }\end{array}$ & $\begin{array}{l}\text { Suggestions and interventions to } \\
\text { improve HRQoL from HF patient's } \\
\text { perspectives. }\end{array}$ \\
\hline
\end{tabular}

\section{References}

[1]. Liu, L, Eisen, H (2014) Epidemiology of heart failure and scope of the problem. Cardiology Clinics Journal 32: 1-8, vii.

[2]. Heidenreich, P,Trogdon, J,Khavjou, O,Butler, J,Dracup, K, et al. (2011) Forecasting the future of cardiovascular disease in the United States: a policy statement from the American Heart Association. Circulation Journal 123: 933-44.

[3]. Huang,Moser, D,Hwang, S,Lennie, T,Chung, M, et al. (2010) Comparison of health-related quality of life between American and Taiwanese heart failure patients. Journal of Transcultural Nursing 21: 212-9.

[4]. Grady,De Leon,Kozak,Cursio,Richardson, et al. (2014) Does self-management counseling in patients with heart failure improve quality of life? Findings from the Heart Failure Adherence and Retention Trial (HART). Quality of Life Research Journal 23: 31-8.

[5]. Vaillant, H,Laporte, C,Pereira, B,Tanguy, G,Cassagnes, J, et al. (2014) Patient education in chronic heart failure in primary care (ETIC) and its impact on patient quality of life: design of a cluster randomised trial. Bio Midecal Central of Family Practice Journal 15: 208.

[6]. Aburuz, M,Alaloul, F,Saifan, A,Masa'deh, R, Abusalem, S (2015) Quality of Life for Saudi Patients With Heart Failure: A CrossSectional Correlational Study. Global Journal of Health Science 8: 45752.

[7]. Inc., Hg. Statistics by Country for Congestive Heart Failure. 2012 [cited 2016 11/9/2016].

[8]. Whoqol Group (1995) The World Health Organization quality of life assessment (WHOQOL): position paper from the World Health Organization. Social science \& medicine Jornal 41: 1403-1409.

[9]. Hamming, J, De Vries, J (2007) Measuring quality of life. British journal of surgery 94: 923-924.

[10]. Hoekstra, T,Lesman, I,Van Veldhuisen, D,Sanderman, R, Jaarsma, T (2011) Quality of life is impaired similarly in heart failure patients with preserved and reduced ejection fraction. European Journal of Heart Failure 13: 1013-8.

[11]. Moh Jordan. Ministry of Health Annual Statistical Book 2014. http://apps.moh.gov.jo 2014 [cited 2015.

[12]. Department of Statistics Jordan. Population in Jordan. http://www.dos.gov.jo 2015 [cited 2016.

DOI: $10.9790 / 1959-0601031421 \quad$ www.iosrjournals.org 
[13]. Brown, D,Mokdad, A,Walke, H,As' Ad, M,Al-Nsour, M, et al. (2009) Projected burden of chronic, noncommunicable diseases in Jordan. Preventing chronic disease 6.

[14]. Zindah, M,Belbeisi, A,Walke, H, Mokdad, A (2008) Obesity and diabetes in Jordan: findings from the behavioral risk factor surveillance system, 2004. Preventing Chronic Disease Journal 5: A17.

[15]. Hunt, S,Baker, D,Chin, M,Cinquegrani, M,Feldman, A, et al. (2001) ACC/AHA Guidelines for the Evaluation and Management of Chronic Heart Failure in the Adult: Executive Summary A Report of the American College of Cardiology/American Heart Association Task Force on Practice Guidelines (Committee to Revise the 1995 Guidelines for the Evaluation and Management of Heart Failure): Developed in Collaboration With the International Society for Heart and Lung Transplantation; Endorsed by the Heart Failure Society of America. Circulation Journal 104: 2996-3007.

[16]. Urden, Ld,Stacy, Km, Lough, Me, Critical Care Nursing, Diagnosis and Management, 7: Critical Care Nursing. 2013: Elsevier Health Sciences.

[17]. Riedinger, M,Dracup, K,Brecht, L, Investigators, S (2002) Quality of life in women with heart failure, normative groups, and patients with other chronic conditions. American Journal of Critical Care 11:211-219.

[18]. Alla, F,Briancon, S,Guillemin, F,Juilliere, Y,Mertes, P, et al. (2002) Self-rating of quality of life provides additional prognostic information in heart failure. Insights into the EPICAL study. European Journal of Heart Failure 4: 337-343.

[19]. Scott, L (2000) Caregiving and care receiving among a technologically dependent heart failure population. Advances in Nursing Science Journal 23: 82-97.

[20]. Sneed, N,Paul, S,Michel, Y,Vanbakel, A, Hendrix, G (2001) Evaluation of 3 quality of life measurement tools in patients with chronic heart failure. Heart \& Lung: The Journal of Acute and Critical Care 30: 332-340.

[21]. Bosworth, H,Steinhauser, K,Orr, M,Lindquist, J,Grambow, S, et al. (2004) Congestive heart failure patients' perceptions of quality of life: the integration of physical and psychosocial factors. Aging \& mental health Journal 8: 83-91.

[22]. Dracup, K,Walden, J,Stevenson, L, Brecht, M (1991) Quality of life in patients with advanced heart failure. The Journal of heart and lung transplantation: the official publication of the International Society for Heart Transplantation 11: 273-279.

[23]. Riegel, B,Moser, Dk,Carlson, B,Deaton, C,Armola, R, et al. (2003) Gender differences in quality of life are minimal in patients with heart failure. Journal of cardiac failure 9: 42-48.

[24]. Van Jaarsveld, C,Sanderman, R,Miedema, I,Ranchor, Av, Kempen, Gi (2001) Changes in Health-Related Quality of Life in Older Patients with Acute Myocardial Infarction or Congestive Heart Failure: A Prospective Study. Journal of the American Geriatrics Society 49: 1052-1058.

[25]. Grady,Jalowiec, A, White, C (1999) Predictors of quality of life in patients at one year after heart transplantation. The Journal of heart and lung transplantation 18: 202-210.

[26]. Rogers, A,Addington, J,Abery, A,Mccoy, A,Bulpitt, C, et al. (2000) Knowledge and communication difficulties for patients with chronic heart failure: qualitative study. British Medical Journal 321: 605-7.

[27]. Chien, C,Lee, C,Wu, Y, Wu, Y (2011) Home-based exercise improves the quality of life and physical function but not the psychological status of people with chronic heart failure: a randomised trial. Journal of Physiotherapy 57: 157-163.

[28]. Chrysohoou, C,Tsitsinakis, G,Vogiatzis, I,Cherouveim, E,Antoniou, C, et al. (2014) High intensity, interval exercise improves quality of life of patients with chronic heart failure: a randomized controlled trial. Quarterly Journal of Medicine 107: 25-32.

[29]. Heo,Doering,Widener, Moser (2008) Predictors and effect of physical symptom status on health-related quality of life in patients with heart failure. Amirecan Journal of Critical Care 17: 124-32.

[30]. Meredith, C,Symonds, P,Webster, L,Lamont, D,Pyper, E, et al. (1996) Information needs of cancer patients in west Scotland: cross sectional survey of patients' views. British Medical Journal 313: 724-726.

[31]. Chung, M,Moser, D,Lennie, T, Frazier, S (2013) Perceived social support predicted quality of life in patients with heart failure, but the effect is mediated by depressive symptoms. Quality of Life Research Journal 22: 1555-63.

[32]. Iqbal, J,Francis, L,Reid, J,Murray, S, Denvir, M (2010) Quality of life in patients with chronic heart failure and their carers: a 3-year follow-up study assessing hospitalization and mortality. European Journal of Heart Failure 12: 1002-8.

[33]. Riegel,Ratcliffe, S,Weintraub, W,Sayers, S,Goldberg, L, et al. (2012) Double jeopardy: the influence of excessive daytime sleepiness and impaired cognition on health-related quality of life in adults with heart failure. European Journal of Heart Failure 14: $730-6$

[34]. Erceg, P,Despotovic, N,Milosevic, D,Soldatovic, I,Zdravkovic, S, et al. (2013) Health-related quality of life in elderly patients hospitalized with chronic heart failure. Journal of Clinical Interventions in Aging 8: 1539-46.

[35]. Eisele, M,Blozik, E,Stork, S,Trader, Jm,Herrmann-Lingen, C, et al. (2013) Recognition of depression and anxiety and their association with quality of life, hospitalization and mortality in primary care patients with heart failure - study protocol of a longitudinal observation study. Bio Medical Central of Family Practice Journal 14: 180.

[36]. Zambroski, Ch,Moser, D,Bhat, G, Ziegler, C (2005) Impact of symptom prevalence and symptom burden on quality of life in patients with heart failure. European Journal of Cardiovascular Nursing 4: 198-206.

[37]. Lewis, E,Lamas, G,O'meara, E,Granger, C,Dunlap, M, et al. (2007) Characterization of health-related quality of life in heart failure patients with preserved versus low ejection fraction in CHARM. European Journal of Heart Failre 9: 83-91.

[38]. Dunderdale, K,Furze, G,Thompson, D,Beer, S, Miles, J (2007) Health-related quality of life from the perspective of patients with chronic heart failure. The British Journal of Cardiology 14: 207 Y212.

[39]. Heo,Lennie, T,Okoli, C, Moser, D (2009) Quality of life in patients with heart failure: ask the patients. Heart and Lung Journal 38: $100-8$.

[40]. Dehkordi, A, Far, A (2015) Effect of exercise training on the quality of life and echocardiography parameter of systolic function in patients with chronic heart failure: a randomized trial. Asian Journal of Sports Medicine Journal 6: e22643.

[41]. Jiang, W,Alexander, J,Christopher, E,Kuchibhatla, M,Gaulden, L, et al. (2001) Relationship of depression to increased risk of mortality and rehospitalization in patients with congestive heart failure. Archives of internal medicine Journal 161: 1849-1856.

[42]. Rietbrock, N,Hamel, M,Hempel, B,Mitrovic, V,Schmidt, T, et al. (2001) [Actions of standardized extracts of Crataegus berries on exercise tolerance and quality of life in patients with congestive heart failure]. Arzneimittel-Forschung journal 51: 793-798.

[43]. Huang,Chung, H,Kroenke, K,Delucchi, K, Spitzer, R (2006) Using the patient health questionnaire-9 to measure depression among racially and ethnically diverse primary care patients. Journal of general internal medicine 21:547-552.

[44]. Lowe, B,Unutzer, J,Callahan, C,Perkins, A, Kroenke, K (2004) Monitoring depression treatment outcomes with the patient health questionnaire-9. Medical care Journal 42: 1194-1201.

[45]. Todero, C,Laframboise, L, Zimmerman, L (2001) Symptom status and quality-of-life outcomes of home-based disease management program for heart failure patients. Outcomes management Journal 6: 161-168.

[46]. Holst, D,Kaye, D,Richardson, M,Krum, H,Prior, D, et al. (2001) Improved outcomes from a comprehensive management system for heart failure. European journal of heart failure 3: 619-625. 
[47]. Wehbe-Alamah, H (2008) Bridging generic and professional care practices for Muslim patients through use of Leininger's culture care modes. Contemporary Nurse Journal 28: 83-97.

[48]. Bui, A,Horwich, T, Fonarow, G (2011) Epidemiology and risk profile of heart failure. Nature Reviews Cardiology 8: 30-41.

[49]. Philbin, E,Dec, W,Jenkins, P, Disalvo, T (2001) Socioeconomic status as an independent risk factor for hospital readmission for heart failure. The American journal of cardiology 87: 1367-1371.

[50]. Al-Krenawi, A, Graham, J (2000) Culturally sensitive social work practice with Arab clients in mental health settings. Health \& Social Work Journal 25: 9-22.

[51]. Hanan, H, Glennon, C (2011) Cultural influences on health care in Palestine. Clinical journal of oncology nursing $15: 281$.

[52]. Brostrom, A,Stromberg, A,Dahlstrom, U, Fridlund, B (2004) Sleep Difficulties, Daytime Sleepiness, and Health-related Quality of Life in Patients With Chronic Heart Failure. Journal of Cardiovascular Nursing 19: 234-242.

[53]. Beery, T,Baas, L,Fowler, C, Allen, G (2002) Spirituality in persons with heart failure. Journal of Holistic Nursing 20: 5-25.

[54]. Silbermann, M, Hassan, E (2011) Cultural perspectives in cancer care: impact of Islamic traditions and practices in Middle Eastern countries. Journal of pediatric hematology/oncology 33: S81-S86.

[55]. Bekelman, D,Hooker, S,Nowels, C,Main, D,Meek, P, et al. (2014) Feasibility and acceptability of a collaborative care intervention to improve symptoms and quality of life in chronic heart failure: mixed methods pilot trial. Journal of Palliative Medicine 17: 14551.

[56]. Rifai, L,Pisano, C,Hayden, J,Sulo, S, Silver, M (2015) Impact of the DASH diet on endothelial function, exercise capacity, and quality of life in patients with heart failure. Proceedings of (Baylor University. Medical Center) 28: 151-6. 